\title{
A Case of Circadian Rhythm Sleep-Wake Disorder in Partial Blindness
}

\author{
Hayom Kim¹, Jinhwan Park², Jung Bin Kim ${ }^{1}$ \\ ${ }^{1}$ Departments of Neurology and ${ }^{2}$ Ophthalmology, Korea University Anam Hospital, Korea University College of Medicine, Seoul, Korea
}

Received May 11,2018

Revised June 8, 2018

Accepted June 12, 2018

Address for correspondence Jung Bin Kim, MD Department of Neurology,

Korea University Anam Hospital, Korea University

College of Medicine,

73 Inchon-ro, Seongbuk-gu,

Seoul 02841, Korea

Tel: $+82-2-920-5510$

Fax: +82-2-920-2472

E-mail: brainbin80@gmail.com
Although it is well known that sleep disturbances can be developed in complete blindness, normally entrained circadian rhythm was observed in the majority of patients with partial blindness. Here, we describe a case with circadian rhythm sleep-wake disorder in partial loss of light perception. A 58-year-old man presented with difficulty in sleep initiation and excessive daytime sleepiness after retinal surgery. The electroretinography revealed partial impairment of light perception in the right side and preserved light perception in the left side. He was diagnosed as circadian rhythm sleep-wake disorder due to impaired light perception. While taking $2 \mathrm{mg}$ of melatonin regularly at 9 every night, his sleep cycle and difficulty in sleep initiation were gradually improved and became fully normalized after 2 weeks. Circadian rhythm sleep-wake disorder could be developed even in partial blindness. Melatonin supplements could effectively improve the circadian rhythm sleep-wake disorder in partial blindness, like as in complete blindness.

J Sleep Med 2018;15(1):31-34

Key Words: Circadian rhythm sleep-wake disorder, Partial blindness, Melatonin, Electroretinography.
Since the biological circadian rhythm is set to a longer than 24 hours (24.2-24.9 hours), entrainment and synchronization are necessary to adapt to the Earth's 24 -hour lightdark cycle. ${ }^{1,2}$ Light is a well-known powerful zeitgeber to entrain and synchronize circadian rhythm to the 24-hour lightdark cycle. ${ }^{1,3}$ Therefore, free running circadian rhythm sleepwake disorder can be developed in patients with complete blindness in association with disruption of light perception. ${ }^{4-6}$ Although it is well known that sleep disturbances can be developed in complete blindness, normally entrained circadian rhythm was observed in the majority of patients with partial blindness. A previous study suggested that entrainment is possible if only some degree of light perception is possible. ${ }^{4}$ However, the association between the degree of impaired light perception and disturbance of entrainment has not yet been clearly described. Here, we describe a case of circadian rhythm sleep-wake disorder that was developed by partial loss of light perception.

This is an Open Access article distributed under the terms of the Creative Commons Attribution Non-Commercial License (https://creativecommons.org/licenses/by-nc/4.0) which permits unrestricted non-commercial use, distribution, and reproduction in any medium, provided the original work is properly cited.

\section{Case Report}

A 58-year-old man presented with difficulty in sleep initiation and excessive daytime sleepiness for the past 6 weeks. Zolpidem $10 \mathrm{mg}$ was administrated by primary physician, however, it was not effective to improve sleep-wake cycle. He was referred to a tertiary university-affiliated hospital with daily sleep diaries completed a week prior to visit. Until 2 months ago, he had operated his shop, woke up at 5 am and slept at midnight on both weekdays and weekends. Based on the sleep diaries, he went to bed at midnight everyday, but sleep onset latency was getting longer, recently he fell asleep at $6 \mathrm{am}$. He complained that he had satisfactory sleep only for two hours a day and had excessive daytime sleepiness, but he did not take naps. Epworth sleepiness scale score was 22, Insomnia Severity Index was 20, and Pittsburgh Sleep Quality Index was 19. He had exudative macular degeneration and retinal detachment. However, he did not have any medical history including hypertension, dyslipidemia, and diabetes. He was a smoker who had been smoking a half a pack of cigarettes per day for 30 years, so smoking was considered as the cause of his ophthalmologic conditions. He did not have any psychiatric history, including mood disorders or anxiety disorders. The Korean version of Beck Depression Inventory-2 score was 8 , depres- 
sion domain score of the Hospital Anxiety-Depression Scale (HADS) was 4, and anxiety domain score of the HADS was 6. He had no family history of sleep disorders including circadian sleep-wake rhythm disorders. He denied any use of substance or medication except for zolpidem. His neurological examination was unremarkable, except for sluggish pupil reflex. Brain MRI revealed no evidence of brain parenchymal structural lesion throughout the visual pathway; however, there was an intraocular hypointense lesion in the right side and distortion of bilateral ocular margin (Fig. 1).

Two months before, he underwent laser surgery for bilateral exudative macular degeneration and retinal detachment. Intraocular silicone oil was injected in the right vitreous space. After the surgery his visual acuity gradually diminished to the degree of 'hand motion' of the semiquantitative scale for visual acuity measurement, which state is capable of light perception but not counting fingers. On the follow-up ophthalmologic examination, electroretinography (ERG) recording revealed an absent of dark-adapted retinal response and scanty light-adapted responses (Fig. 2) in the right side. Both dark- and light-adapted ERG recordings in the left side were within normal limit (Fig. 2). These ERG findings suggest partial impairment of light perception in the right side and preserved light perception in the left side.

He was diagnosed as circadian rhythm sleep-wake disorder, based on the clear temporal relationship between opthalmologic surgery with impared light perception and sleep distur-

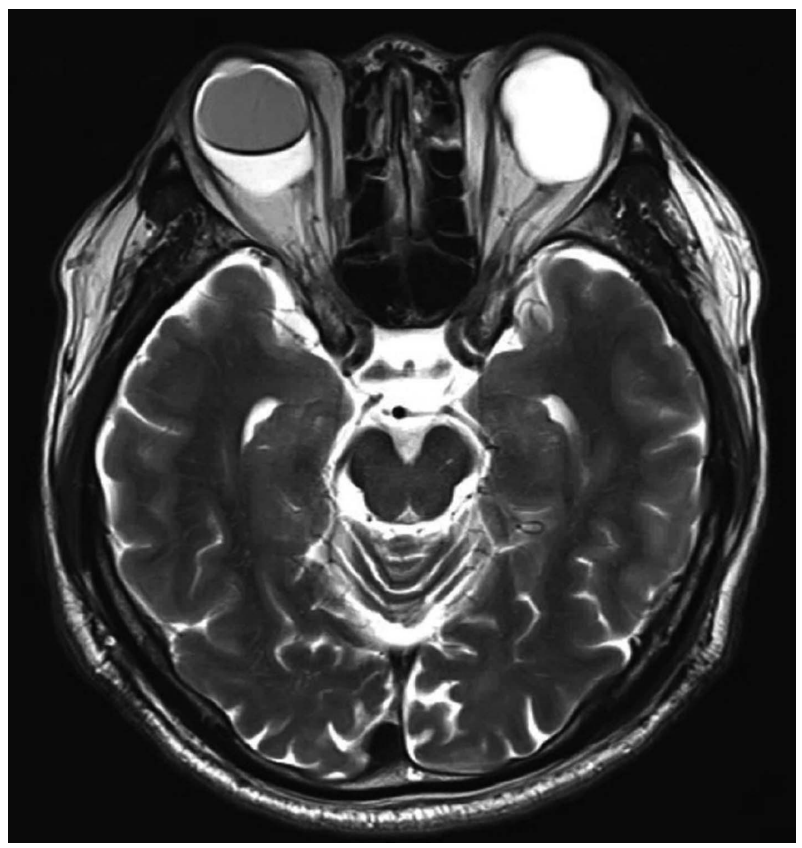

Figure 1. The axial T2-weighted MRI shows an intraocular low signal intensity lesion (injected silicone oil) in the right side and distortion of bilateral ocular margin. There is no brain parenchymal lesion throughout the visual pathway. bance. He was prescribed to take $2 \mathrm{mg}$ of melatonin regularly at 9 every night. While taking the melatonin regularly, his sleep cycle and difficulty in sleep initiation were gradually improved and became fully normalized after 2 weeks. Melatonin was maintained for a total of 1 month. After discontinuation of melatonin, he had no sleep disturbance for 3 months.

\section{Discussion}

It has been reported that free running circadian sleep disorder could be developed in complete blindness. ${ }^{4-6}$ The proposed pathophysiological mechanism regarding the circadian rhythm sleep-wake disorder in complete blindness is blockage of environmental zeitgeber (i.e., bright light) through retinohypothalamic tract caused by disruption of light perception. ${ }^{2}$ The blockage of signal transmission leads to reduced melatonin secretion and ultimately disrupts the circadian rhythm.

There are several lines of evidence that melatonin levels were reduced, and melatonin supplements improved free running circadian sleep disorder in patients with complete blindness. To our best knowledge, however, it is unclear which degree of impaired light perception can deteriorate the circadian rhythm. A previous study showed that blind subjects with some degree of light perception mainly have normally entrained circadian rhythms, suggesting that only a small intensity of light might be required to maintain the normally entrained circadian rhythm. ${ }^{4}$ On the contrary, our case showed circadin rhythm sleep disorder can be seen in partial blindness. Clear temporal relationship between the opthalmologic surgery and circadian dysregulation as well as effectively compensation with melatonin (a potential endogenous zeitgeber) supplements suggest that circadian rhythm sleep-wake disorder can be seen in partial blindness. The underlying mechanism of differences in the susceptibility to sleep dysregulation in response to impaired light perception between the previously reported cases and ours is not clear. However, since the input pathway carrying information about light-dark cycle to the suprachiasmatic nucleus through retinohypothalamic tract starts in a particular type of retinal ganglion cells containing melanopsin (i.e., intrinsically photosensitive retinal ganglion cells), differences in the degree of selectively disruption of these cells may be the possible explanation for the differences in the susceptibility. ${ }^{8}$ In addition, difference in the contributing amount of nonphotic zeitgeber, such as eating and physical activity between the subjects may be another explanation.

The ERG is a useful test that can demonstrate the functions of retina by recording the electrical responses to optical stimulations from various cells distributed in the retina. The waveforms recorded on the retinal electric potential by optical stimuli have respective sources. The first negative poten- 
Right

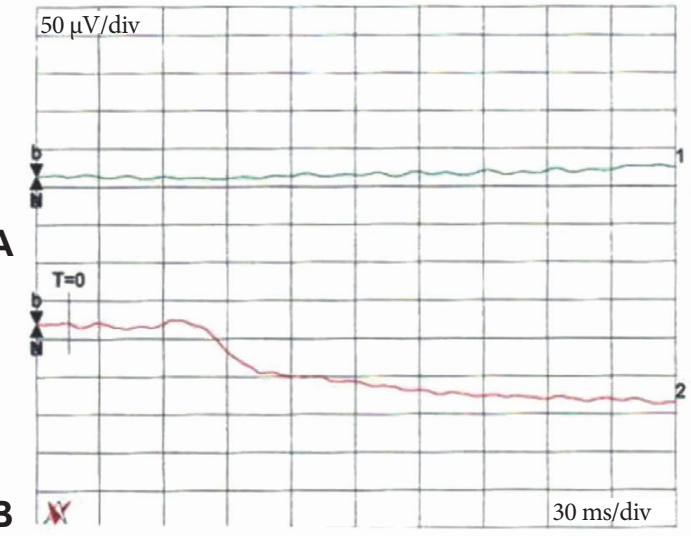

Skot. ERG

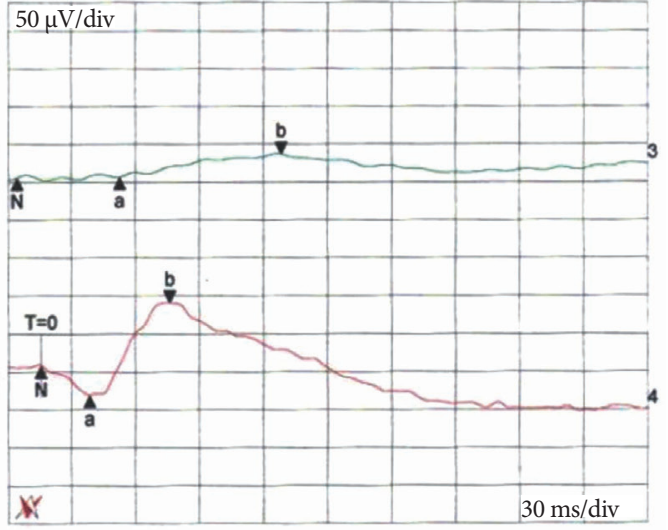

Oscillatory-P.

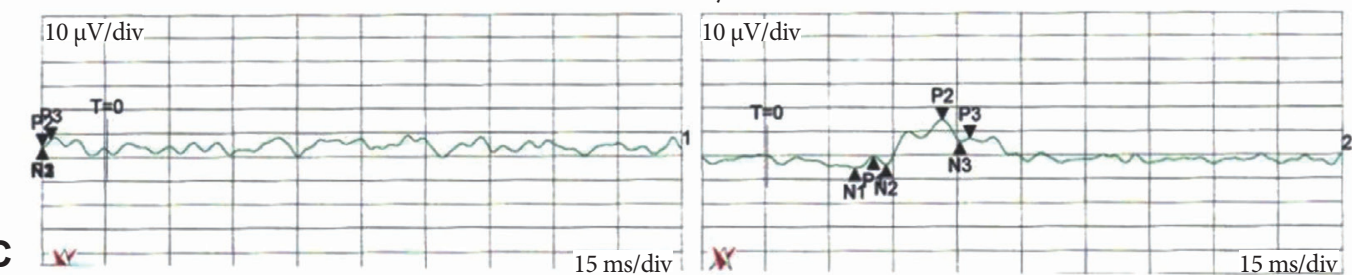

Phot. ERG

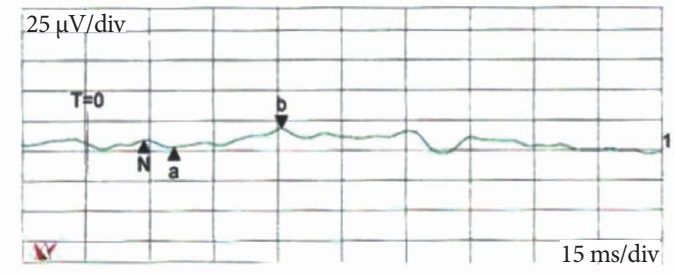

$30 \mathrm{~Hz}$ flicker
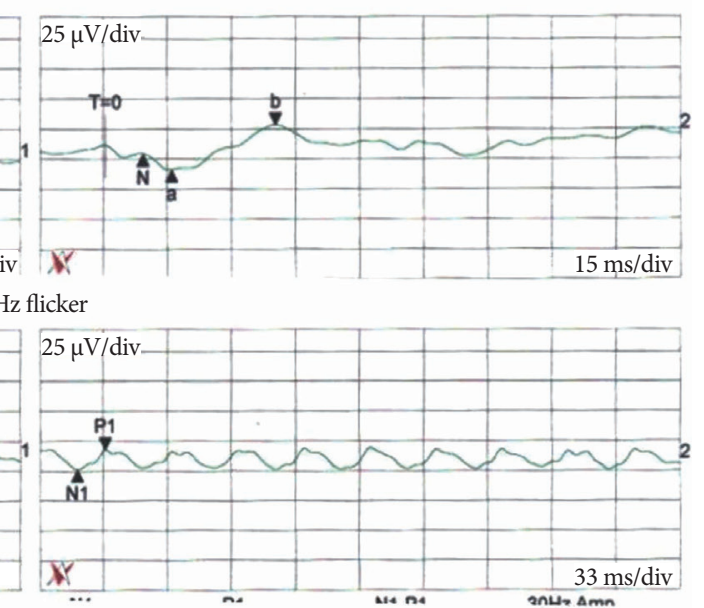

Figure 2. Dark-adapted ERG recording reveals an absent of rod response $(A)$ in the right side and combined rod-cone responses (B) in the right side. (C) Oscillatory potential is not evoked in the right side. Light-adapted ERG recordings show an attenuated single flash cone response (D) and scanty response to the $30 \mathrm{~Hz}$ flicker (E) in the right side. Both dark- and light-adapted ERG recordings in the left side are within normal limit. ERG: electroretinography.

tial, a wave, refers to the potential of the photoreceptor including rod cell and cone cell. The positive $b$ wave is generated by the bipolar cells and the Müller cells. The oscillating potentials are composed of 3-4 small potentials that represent the signal transmission in the inner retina. By interpreting the distinct patterns of the a- and b-waves under dark-adapted and light-adapted conditions, information about localization of the cause of visual loss could be acquired. It has been known that ERG can sensitively assess the retinal function even in early stage of macular degeneration. ${ }^{9}$ Moreover, given that ERG can be used to quantitatively evaluate the functional integrity of the retinohypothalamic tract, ${ }^{10}$ the ERG record- ings might be a useful supportive tool for diagnosing circadian rhythm sleep-wake disorder induced by impaired light perception. We did not perform melatonin level testing before and after the treatment in this case; therefore, directly quantitative measurements of decreased melatonin level due to impaired light perception and improvement of melatonin level after the treatment were limited. Further longitudinal study using ERG recordings for quantitative measurement of the degree of impaired light perception and its relationship with melatonin level might provide important information about establishing a therapeutic strategy in circadian rhythm sleep -wake disorder in association with partial blindness. 
In summary, even partial blindness can have circadian rhythm sleep-wake disorder. Melatonin supplements could effectively improve the circadian rhythm sleep-wake disorder in partial blindness, like as in complete blindness. The ERG recordings can objectively quantify the degree of impairment of light perception. The quantitative information about the relationship between the light perception and the melatonin level might be useful in diagnosing and establishing a therapeutic strategy in circadian rhythm sleep-wake disorder due to impaired light perception.

\section{REFERENCES}

1. Duffy JF, Wright KP Jr. Entrainment of the human circadian system by light. J Biol Rhythms 2005;20:326-338.

2. Saper CB, Scammell TE, Lu J. Hypothalamic regulation of sleep and circadian rhythms. Nature 2005;437:1257-1263.

3. Cajochen C. Alerting effects of light. Sleep Med Rev 2007;11:453-464.
4. Skene DJ, Arendt J. Circadian rhythm sleep disorders in the blind and their treatment with melatonin. Sleep Med 2007;8:651-655.

5. Quera Salva MA, Hartley S, Léger D, Dauvilliers YA. Non-24-hour sleep-wake rhythm disorder in the totally blind: diagnosis and management. Front Neurol 2017;8:686.

6. Lockley SW, Arendt J, Skene DJ. Visual impairment and circadian rhythm disorders. Dialogues Clin Neurosci 2007;9:301-314.

7. Czeisler CA, Shanahan TL, Klerman EB, et al. Suppression of melatonin secretion in some blind patients by exposure to bright light. $N$ Engl J Med 1995;332:6-11.

8. Bonmati-Carrion MA, Arguelles-Prieto R, Martinez-Madrid MJ, et al. Protecting the melatonin rhythm through circadian healthy light exposure. Int J Mol Sci 2014;15:23448-23500.

9. Feigl B, Brown B, Lovie-Kitchin J, Swann P. Monitoring retinal function in early age-related maculopathy: visual performance after 1 year. Eye (Lond) 2005;19:1169-1177.

10. Pérez-Rico C, de la Villa P, Arribas-Gómez I, Blanco R. Evaluation of functional integrity of the retinohypothalamic tract in advanced glaucoma using multifocal electroretinography and light-induced melatonin suppression. Exp Eye Res 2010;91:578-583. 